\title{
Where is the ball? \\ Behavioral and neural responses elicited by a magic trick
}

Hugo Caffaratti a , Joaquin Navajas ${ }^{\text {a,b }}$, Hernan G. Rey ${ }^{\text {a }}$, and Rodrigo Quian Quiroga ${ }^{\mathrm{a}, *}$

${ }^{a}$ Centre for Systems Neuroscience, University of Leicester, 9 Salisbury Road, LE1 7QR, United Kingdom

${ }^{\mathrm{b}}$ Institute of Cognitive Neuroscience, University College London, 17 Queen Square, London, WC1N 3AR, UK

${ }^{*}$ Corresponding Author

Centre for Systems Neuroscience

University of Leicester

9 Salisbury Road

LE1 7QR

Leicester

United Kingdom

rqgg1@le.ac.uk 


\section{Abstract}

We present results from two experiments where subjects watched continuous videos of a professional magician, repeatedly performing a maneuver in which a ball could "magically" appear under a cup. In all cases, subjects were asked to predict whether the ball would appear under the cup or not, while scalp EEG recordings were performed. Both experiments elicited strong and consistent behavioral and neural responses. In the first experiment, we used two blocks of videos with different probabilities of the ball appearing in the cup and found that: first, based on the behavioural responses, the subjects could track this probability change and second, the different probabilities modulated the neural responses. In the second experiment, we introduced a control condition in which the magician performed the maneuver under the table, out of subjects' view. Comparing the two conditions (i.e. performing the maneuver under or out of the subjects' view), we found that: first, the magic trick dramatically biased the subjects' behavioral responses, and second, the two conditions led to differential neural responses, in spite of the fact that the stimulus triggering the evoked responses (seeing the ball in the cup) was exactly the same. Altogether, our results show how new insights into sensory and cognitive processing can be obtained using adapted magic tricks. Moreover, the approach of analyzing responses to continuous video presentations offers a more ecological setting compared to classic evoked potential paradigms, based on presenting static images flashed at the center of the screen.

\section{Introduction}


It has long been recognized that, from relatively limited, noisy and ambiguous information, perception relies on unconscious inferences based on previous experiences (Helmholtz, 1878a; Helmholtz, 1878b; Gregory, 1973). For thousands of years magicians have been aware of this fact and have learnt how to manipulate perception by breaking these assumptions at will. Although the scientific interest in magic is not new (Binet and Nichols, 1896; Jastrow, 1897; Triplett, 1900), in the last decades there has been an increasing interest in setting up parallels between magic theory and cognitive neuroscience, in some cases leading to the adaptation of magic tricks into new experimental paradigms (Cui et al., 2011; Rieiro et al., 2013; OteroMillan et al., 2011; Johansson et al., 2005; Kuhn and Land, 2006b; Macknik et al., 2008; Martinez-Conde and Macknik, 2008; Parris et al., 2009; Kuhn and Findlay, 2010; Olson et al., 2012; Shalom et al., 2013; Quian Quiroga, 2016; Lamont and Wiseman, 2005; Kuhn et al., 2014). Most of these experiments have focused on the subjects' behavioral and oculomotor responses while subjects watched magic tricks. However, only very few studies have dealt with brain responses elicited by magic tricks, localizing areas that contrasted responses upon different conditions with fMRI recordings (Parris et al., 2009; Danek et al., 2015) It is within this context that we set out to study whether the observation of a magic trick might trigger specific patterns of brain activations, as measured with scalp EEG. To address this question, we recorded EEG signals of subjects watching videos of an adaptation of a famous magic trick, The Chop-Cup (Mark Wilson \& Earl Nelson, 1979), in which a ball taken from a cup may "magically" reappear inside it (a similar type of trick has been used in Riero et al (2013)). We hypothesized that the unexpected appearance of the ball in the cup compared to trials where the ball does not appear in the cup, as expected - will trigger a P3 response as the one observed upon oddball paradigms, where an unexpected 
stimulus (the target stimulus triggering the P3 response), is presented randomly and sporadically among a sequence of non-target stimuli (Sutton et al., 1965). In other words, we used the classic P3 response as a marker of the subjects' reactions upon observing the ball magically appearing (or not appearing) inside the cup. A main reason for choosing this trick is that it can be repeated many times without the subjects noticing how it is done, thus allowing calculating ensemble averages of several trials to obtain reliable neural responses. Furthermore, given that subjects were presented videos showing continuous repetitions of the magician performing (or not) the trick, this experiment allowed us to characterize brain responses obtained in a much more natural condition compared to the classic evoked potential paradigms implemented by flashing images, which require subjects to keep their fixation at the center of the screen (Luck and Kappenman, 2011; Freeman and Quian Quiroga, 2012).

Given the sequence of trials presented in the video, we further hypothesized that the strength of this P3-like response should be modulated by the probability of appearance of the unexpected stimuli (ball in the cup), as described with classic oddball paradigms (Polich, 2007). To test these hypotheses, in a first experiment (Experiment 1) subjects saw two blocks of videos of sequential trials, in which the manipulation of the ball and the cup was performed under the subjects' view (Direct Load condition, see Methods). The probability of the ball appearing in the cup was $50 \%$ in the first block and $30 \%$ in the second block. The rationale for using a 50/50 probability was to test if, even with equal probability, the appearance of the ball in the cup will still give a P3-like response due to the fact that this "magical" event is unexpected. In a second experiment (Experiment 2), we introduced a control condition (Indirect Load condition, see Methods), in which the movement of 
removing the ball from the cup (or not) was performed under the table, out of the subjects' view. The rationale for introducing this condition, in which the eventual appearance of the ball in the cup cannot be attributed to the performance of a magic trick, was to test the hypothesis that the same stimulus (ball in the cup) may trigger different brain responses when appearing as a result of a magic effect (Direct Load) or not (Indirect Load). More generally, our goal was to explore the possibility of adapting a magic trick into a new paradigm in cognitive neuroscience that gives differential brain responses from exactly the same stimulus, as it has been described with binocular rivalry, flash suppression, background masking or the morphing of the stimuli, among others (Quian Quiroga et al., 2014; Quian Quiroga et al., 2008; Logothetis, 1998; Blake and Logothetis, 2002; Kanwisher, 2001; Navajas et al., 2013) Altogether we found that the video presentations of the magic trick elicit strong evoked potentials, similar to the ones described in classic oddball paradigms. These evoked potentials, as well as the behavioural responses by the subjects, were modulated both by the probability of the stimuli and by the performance of the magic trick.

\section{Materials \& Methods}

\subsection{Subjects}

A total of 35 participants (24 females; 26 right-handed; mean age 25.6; range 19-40 years old) volunteered for this study. All of them had normal or corrected-to-normal vision, and no history of neurological disorders. Ten subjects participated in 
Experiment 1 and the remaining 25 participated in Experiment 2. Data from 5 other participants were rejected due to an excessive number of artifacts in the EEG recordings.

\subsection{Stimuli}

Participants observed videos projected on a CRT monitor. The screen resolution was $1024 \times 768$ pixels, the refresh rate $100 \mathrm{~Hz}$ and the viewing distance was approximately $55 \mathrm{~cm}$. The stimuli consisted of videos of a professional magician (H.C.) repeatedly performing a magic trick (see Paradigm for details). Videos were filmed using a standard video camera mounted on a tripod with a sampling frequency of 25 frames/sec. The presentation of the videos (without sound) was controlled and synchronized to the EEG recording system using MATLAB (Mathworks, Natick, MA) and the Psychophysics toolbox (Brainard, 1997).

\subsection{Paradigm}

Each video comprised a sub-block of 50 consecutive trials, in which an adapted version of the "Chop Cup" magic trick was repeatedly shown (Wilson and Nelson, 1979). On each trial, the magician inserted a red ball into a metal cup, turned the cup upside down while covering its mouth with the other hand, which then executed a movement that pretended to remove the ball from the cup. Immediately after this movement, the cup was maintained upside down in the air for few seconds before placing it on the table (thus being very surprising that the ball could be inside the 
cup). We call these sequence of movements 'Direct Load'. (see Figure 1 (A) and Movie 1). At that time, the video was automatically paused and the subjects were instructed to guess whether the ball was in the cup or in the magician's hand, using the left and right arrow keys, respectively. After a short delay following the subjects' responses (see below) the video was restarted, showing either that the ball was not there (i.e. it was retained in the magician's hand, as one would expect), or that it "magically" appeared under the cup. For each trial, we considered the stimulus onset to be the first video frame at which the experimenter started to tilt the cup to show its content.

In Experiment 1, participants underwent two consecutive Direct Load blocks, consisting of 250 trials each (i.e. 5 sub-blocks of 50 trials). In the first block, the probability of the ball appearing "magically" inside the cup was 50\%, whereas in the second block this probability was $30 \%$. The two blocks were separated by a 5-10 min break. For this experiment, the video was always restarted $500 \mathrm{~ms}$ after the subjects' decisions, at a point that was exactly 12 frames $(480 \mathrm{~ms})$ before the stimulus onset. These delays were introduced in order to avoid contaminations of the EEG responses due to hand movements in the first case, and the restarting of the video in the latter case. The total duration of the experiment was approximately $90 \mathrm{~min}$.

Experiment 2 consisted of 400 trials presented in 8 sub-blocks of 50 trials each. Throughout this experiment, the probability of the ball appearing inside the cup was $30 \%$. Half of the trials were conducted as in Experiment 1 ('Direct Load' condition), and in the other half of the trials the magician manipulated the ball and the cup under the table out of subjects' view. We will refer to this sequence of movements as 'Indirect Load' (see Figure 1 (B)). This way, the appearance of the ball in the cup was not related to a 'magical effect' but rather to the regular movement of 
putting (or not putting) the ball inside the cup under the table (see movie 2 in Supplementary Material). Altogether, we had 4 conditions:

- Cup_Direct-Load: The magician placed the ball inside the cup and directly manipulated the cup, under subjects' view, clearly pretending to remove the ball. The ball, however, "magically" appeared in the cup. Note that this is the unique condition that we considered to be a magic effect.

- Hand_Direct-Load: The magician repeated the same maneuver, as before, but the cup appeared empty, as expected, given that the ball was retained in the magician's hand.

- Cup_Indirect-Load: The magician placed the ball inside the cup, indirectly manipulated the ball and cup under the table and finally showed that the ball was in the cup.

- Hand_Indirect-Load: The magician performed the same indirect load, as above, but then showed that the cup was empty.

In Experiment 2, trials corresponding to the 'Direct Load' and 'Indirect Load' conditions were randomly interleaved and presented with equal probability (see Movie 2). Due to the fact that in Experiment 1 we observed an EEG response elicited by the restart of the video (see Results section), in Experiment 2 we randomized the time at which the video was paused (between $280 \mathrm{~ms}$ and $680 \mathrm{~ms}$ before stimulus onset, corresponding to 7 and 17 frames, respectively). The total duration of the Experiment was approximately $70 \mathrm{~min}$.

\subsection{EEG Recordings}


EEG data was recorded with 64 scalp electrodes mounted in an elastic cap (10/20 system), using a Biosemi Active-Two System with a sampling frequency of $256 \mathrm{~Hz}$ and a data acquisition bandpass filter between $0.001-100 \mathrm{~Hz}$ to avoid aliasing. The linked bilateral mastoids were used as reference and electrode impedances were kept below $5 \mathrm{~K} \Omega$. For each electrode, $2000 \mathrm{~ms}$ epochs were extracted from the continuous EEG data, $1000 \mathrm{~ms}$ before and $1000 \mathrm{~ms}$ after stimulus onset. Epochs were linearly detrended and baseline corrected. Trials with eye blinks, eye movements, and other artifacts were removed by visual inspection of electrooculogram (EOG) channels. We report results for the midline electrodes $\mathrm{Fz}, \mathrm{Cz}, \mathrm{Pz}$, and $\mathrm{Oz}$.

\subsection{Data analysis}

We focused on the analysis of the P3 responses across different conditions. For a better identification of the single subjects' P3 peak amplitudes and latencies, the average evoked potentials for each subject (and in each condition) were denoised as in previous works (Quian Quiroga, 2000; Ahmadi and Quian Quiroga, 2013). This was achieved by doing a wavelet transform of the signal, selecting a number of wavelet coefficients that were correlated with the evoked responses and then reconstructing the signal (using the inverse wavelet transform) but only from the selected coefficients. For each subject the set of wavelet coefficients chosen for denoising were the same for all conditions. From the denoised traces, the P3 peak amplitude and latency were identified as the maximum in the post stimulus time window $[250,750]$ ms. 
Pairwise statistical comparisons were performed using paired non-parametric Sign tests, with a Bonferroni correction for multiple comparisons.

\section{Results}

\subsection{Behavior}

In Experiment 1 we assessed how the behavioral choice was influenced by the percentage of trials in which the ball appeared in the cup $(50 \%$ in the first block and $30 \%$ in the second block). The left panel of Figure 2 shows the average percentage (across subjects) of 'cup responses' in each block. This proportion was smaller in the second block compared to the first block, suggesting that subjects could track the change in the proportion of 'Cup_Direct-Load' trials (i.e. with the ball appearing inside the cup), although this difference did not reach statistical significance $(p=0.17$; Sign test). Moreover, considering each block separately, the mean percentage of cup responses in the first block (42.8\%) was significantly smaller than the actual proportion of 'Cup_Direct-Load' trials shown (50\%; $\mathrm{p}<0.05$; Sign-test). This lower proportion of 'cup responses' could be attributed to the unexpected (magic) effect of seeing the ball in the cup. The number of 'cup responses' in the second block, $31.9 \%$, was slightly larger though not statistically different from the actual percentage of cup responses shown $(30 \%$; $=0.58$; Sign test). The slight increase in the number of cup responses (rather than a decrease, as in the first block) is likely due to the fact that, during the second block, subjects were still adapting their response pattern from the higher percentage of 'Cup_Direct-Load' trials shown in the first block. 
The right panel of Figure 2 shows the percentage of 'cup responses' in Experiment 2. Even though the probability of the ball appearing in the cup was $30 \%$ for both the 'Direct Load' and 'Indirect Load' conditions, the percentage of 'cup responses' was significantly lower for the 'Direct Load trials' $\left(\mathrm{p}<10^{-5}\right.$; Sign test). As before, the percentage of 'cup responses' was lower than the actual proportion of 'Cup_Direct-Load' trials (30\%; $\mathrm{p}<10^{-3}$; Sign test), but when the movement was done under the table (the 'Indirect Load' condition) the subjects were equally likely to answer either way and the percentage of 'cup responses' was no different from $50 \%$ (Sign test), and it was significantly larger than $30 \%\left(\mathrm{p}<10^{-7}\right.$; Sign test).

Summarizing the behavioral results of both experiments, subjects were able to track changes of the probability of the ball appearing in the cup. In trials where the magic trick was performed (Cup_Direct-Load), subjects had a strong bias towards predicting that the ball was not in the cup, as would naturally be expected, in spite of the fact that they saw the same manipulation performed hundreds of times. In contrast, for the 'Indirect-Load' condition the subjects' responses were 50/50, although the proportion of 'Cup_Indirect-Load trials' was 30\%, as in the 'Direct Load condition'. We argue that since both types of trials (Direct Load and 'Indirect Load') were interleaved, subjects did not keep track separately of the proportion of trials in each condition and compensated downwards in one case (\% of 'cup responses' in the 'Direct Load' condition) and upwards in the other (\% of 'cup responses' in the 'Indirect Load' condition).

\subsection{Neural Responses for experiment 1}


Figure 3 shows the grand average neural responses in the midline electrodes for the two blocks of Experiment 1. We first observe that the video presentations elicited strong evoked responses in all conditions. In particular, we observe a slow negative shift preceding stimulus onset, most pronounced in the central-parietal electrodes. This corresponds to the Contingent Negative Variation (CNV), which has been largely studied in classic evoked potential paradigms and reflects the expectation produced by the anticipation of the stimulus (in our case the revelation of whether the ball was in the cup or not) (Walter et al., 1964). Given that we did not observe differences in the CNV responses across conditions, we did not analyze this response any further. Approximately $200 \mathrm{~ms}$ prior to stimulus onset, a positive deflection is also observed in all conditions, which is due to resuming the video after the pause (this response disappeared when introducing a variable restart time in Experiment 2).

After stimulus onset, all conditions triggered a large positive response, resembling the $\mathrm{P} 3$ evoked potential described in classic oddball paradigms (Sutton et al., 1965; Picton, 1992; Polich, 2007). In particular, in block 2 this response was larger for the less frequent 'Cup_Direct-Load' trials (30\%) compared to the 'Hand_Direct-Load' trials (70\%). Moreover, the amplitude difference between the 'cup' and 'hand' responses was larger in block 2 compared to block 1, where the proportion of cup and hand trials were both $50 \%$ (for all electrodes $\mathrm{p}<0.05$, except $\mathrm{Oz}$ with $\mathrm{p}=0.08$; Sign Test). In addition, and in line with previous evidence (Sutton et al., 1965; Picton, 1992; Polich, 2007), the P3 response to the infrequent 'Cup_DirectLoad' trials in block 2 was preceded by a negative N2 deflection. Finally, we notice an earlier response onset for 'Cup_Direct-Load' compared to 'Hand_Direct-Load' trials, something that we studied further in Experiment 2 with a larger number of subjects. 


\subsection{Neural Responses for Experiment 2}

In Experiment 2 we generated a different set of videos, where in half of the trials (interleaved) the magician manipulated the cup's content under the table: 'Indirect Load' condition (see Methods). The rationale for introducing this condition was to compare responses to the exact same stimulus eliciting the electrophysiological responses (ball in the cup) but dissociating between the 'Direct Load' and 'Indirect Load' conditions (see Methods). Figure 4a shows the grand average responses. As before, we observe a slow negative (CNV) deflection preceding stimulus onset, but in this case, the pre-stimulus responses to the restarting of the video were absent due to the randomization of the restart time (see Methods).

After stimulus onset, we again observe a clear positive deflection, which was significantly larger (for all electrodes $\mathrm{p}<0.005$; Sign test) and earlier (for all electrodes $\mathrm{p}<0.05$, except $\mathrm{Pz}$ with $\mathrm{p}=0.17$; Sign test) for the 'cup trials' compared to the 'hand trials'. The amplitude difference can be attributed to the less likely outcome of finding the ball under the cup ( $30 \%$ of the trials) and the earlier responses for the 'cup trials' are due to a better-defined onset of seeing the ball compared to noticing its absence.

Next, we focused on comparing the 'Direct Load' and 'Indirect Load' conditions. For the 'Hand trials', no significant differences between these two conditions were found, either in the amplitude or in the latency of the P3, which is expected given that the 'magic effect' took place only when the ball appeared in the cup (in the 'Direct Load' condition). For the 'cup trials', there were no significant differences in the amplitude of the P3 response between 'Direct Load' and 'Indirect Load' conditions $(\mathrm{p}=\mathrm{n} . \mathrm{s}$. for all the electrodes; Sign test). However, there was a 
significant delay (of about $50 \mathrm{~ms}$ ) in the latency of the 'cup responses' under the 'Direct Load' condition (for all electrodes $\mathrm{p}<10^{-5}$; Sign test).

The latency difference between the 'Direct Load' and 'Indirect Load' conditions for the 'cup trials' cannot be attributed to the different retinal stimulation, because in this case the visual stimulus (i.e. the red ball appearing in the cup) was the same. However, this difference could in principle be attributed to the different number of correct responses in the 'Direct Load' and 'Indirect Load' conditions, considering that: i) on average, correct responses elicited a significantly earlier response onset compared to incorrect responses (for all electrodes $\mathrm{p}<0.05$; Sign test), and ii) for the 'cup trials' subjects had a larger number of correct responses in the 'Indirect Load' condition (59\%) compared to the 'Direct Load' condition (17\%), given their tendency to answer 'cup' more frequently in the first case (see Figure 2b). To rule out this potential confound, we performed a 2-way ANOVA for the P3 latency of the 'cup trials' with factors: 'condition' (two levels: 'Direct Load' and 'Indirect Load') and 'response' (two levels: 'correct' and 'incorrect'). Latency differences were significant for factor 'condition' ( $\mathrm{p}<0.05$; in all electrodes except $\mathrm{Oz}$ ) and 'response' $(\mathrm{p}<0.05$; in all electrodes except Fz). The interaction between factors was not significant in any of the electrodes, thus showing that these were two independent effects. To further ensure this was the case -i.e. that the latency differences between the 'Direct Load' and 'Indirect Load' conditions were not due to a different number of correct trials we evaluated the latencies for each response type separately (Figure $4 b$ ). The P3 peak latencies for the 'cup trials' occurred significantly later in the 'Direct Load' condition compared to the 'Indirect Load' condition, both when considering only the correct responses (in all cases $\mathrm{p}<0.01$, except $\mathrm{Oz}$ with $\mathrm{p}=0.13$; Sign test) and when considering only the incorrect responses (in all cases $\mathrm{p}<0.05$; Sign test). Overall, these 
results indicate that the perception of the magic action delayed the neural response onset, regardless of whether the trial was correct or incorrect.

\section{Discussion}

In the last few years, several works have made parallels between synergetic theories developed by magicians and neuroscientists (Cui et al., 2011; Kuhn et al., 2008; Lamont and Henderson, 2009; Macknik et al., 2008; Otero-Millan et al., 2011; Rieiro et al., 2013; Quian Quiroga, 2016). Along this line, a handful of works have started to show insights into how magic tricks can be translated into novel neuroscience paradigms (Rieiro et al., 2013; Otero-Millan et al., 2011; Cui et al., 2011; Johansson et al., 2005; Kuhn and Land, 2006a; Macknik et al., 2008; Martinez-Conde and Macknik, 2008; Parris et al., 2009; Kuhn et al., 2010; Olson et al., 2012; Shalom et al., 2013; Raz and Zigman, 2001), in a late revival of pioneering studies performed at the end of the XIX century (Jastrow, 1897; Triplett, 1900; Binet and Nichols, 1896). Within this context, we adapted a known magic trick — the Chop Cup trick, where, after a magic movement, a ball may or may not appear in a cup — to study behavioral decisions made by the subjects as well as their brain evoked responses. Of particular relevance was the fact that the trick was repeatedly shown in a non-edited video, so that subjects could perform a meaningful estimation of the probability of finding the ball in the cup. Related to our approach, videos showing the repeated execution of a related trick involving cups and balls under different conditions were used to study behavioral and oculomotor responses in a recent study (Rieiro et al., 2013).

The study of behavioral and evoked responses generated by the observation of 
the Chop Cup magic trick allowed us to establish three main points. First, we showed that electrophysiological responses could be obtained while subjects freely gaze at a sequence of events shown in a continuous unedited video, in contrast to standard paradigms using images flashed in the center of the screen (where the subjects are asked to withhold fixation). Second, we showed that behavioral and neural responses were biased according to the particular sequence of events shown in the videos - i.e. the probability of observing the ball in the cup following direct and indirect loads. Third, we found that the same stimulus (seeing the ball in the cup) gave differential responses depending on whether this was the result of a magic trick being performed or the natural outcome of a manipulation performed outside the subjects view. With regards to this latter point, we propose that the comparison between these two conditions contributes to the interesting literature in cognitive neuroscience separating between sensory processing and subjective perception, by describing differential neural responses to presentations of the same stimuli (Quian Quiroga et al., 2014; Quian Quiroga et al., 2008; Navajas et al., 2013; Logothetis, 1998; Kanwisher, 2001; Blake and Logothetis, 2002).

In two separate experiments we found clear behavioral and electrophysiological responses. With respect to behavior, in the first experiment we observed that the subjects could track a change in the proportion of 'cup trials' (i.e. trials in which the ball appeared in the cup) between two different blocks. Moreover, in the first block the percentage of 'cup responses' was significantly lower than the presented rate (50\%), a bias that can be attributed to the unexpected effect of seeing the ball in the cup (due to the performance of the magic trick 'Cup_Direct Load'). In the second block, the number of cup responses was slightly higher, though not significantly different, than the presented rate of cup trials (30\%), an effect that is 
likely due to an adaptation from the higher presentation rate of cup trials in the previous block.

The most interesting behavioral results were observed in Experiment 2, where we introduced a control 'Indirect Load' condition, in which the movement of taking the ball out of the cup (or not) was performed under the table. In this case, the number of 'cup responses' was significantly lower for the 'Direct Load' compared to the 'Indirect Load' condition, even though the number of times the ball appeared under the cup was exactly the same in both conditions. Altogether, the behavioral results show that: first, the subjects were able to track changes in the rate of presentation of the different stimuli; and second, that the performance of the magic trick (though repeated hundreds of times) was effective in biasing their responses.

With the electrophysiology recordings we observed a slow negative deflection preceding the stimulus onset (the time of showing the content of cup), which corresponds to the CNV potential observed in classic evoked potential tasks (Walter et al., 1964) and reflects the expectation of an impending stimulus. After stimulus onset, we also observed large evoked responses that resemble the N2-P3 complex elicited with standard oddball paradigms. This response varied with the probability of the unexpected stimulus, as has been described in previous works (Picton, 1992; Polich, 2007).

In Experiment 2, when comparing the 'Direct Load' and 'Indirect Load' conditions we observed delayed neuronal responses upon performance of the magic trick, in spite of the fact that the visual stimulus triggering the responses (seeing the ball in the cup) was exactly the same for both conditions. We also showed that this difference was not due to the different number of correct responses in each condition but could instead be attributed to the fact that subjects needed more time to process 
the unexpected outcome of seeing the ball in the cup after the performance of the magic trick - considering that the expected outcome is that the ball remains in the hand (otherwise it should have fallen out when turning the cup upside down).

It could in principle be argued that the differential evoked responses we observed upon the performance of the magic trick could just be due to surprise or novelty (i.e. irrespective of any specific effect elicited by the magic trick) due to the low probability of occurrence of these trials. This alternative explanation is particularly supported by the fact that surprise has been a main factor described to modulate the $\mathrm{P} 3$ responses in oddball paradigms (Donchin, 1981; Polich, 2007). We can, however, rule out this confound given that in Experiment 2 subjects saw the trick (Cup_Direct-Load trials) being performed a total of 60 times. Moreover, the probability of the ball appearing in the cup in the 'Direct Load' condition (Cup_Direct Load trials) was exactly the same as in the 'Indirect Load' condition (Cup_Indirect Load trials). So, in spite of the fact that subjects have seen the trick several times, breaking natural inferences still led to differential brain responses.

The study of responses to video presentations shows interesting new insights compared to classic evoked potential paradigms (Luck and Kappenman, 2011; Freeman and Quian Quiroga, 2012). Clearly, we see objects continuously moving in our environment and the standard study of responses to presented images is a limited proxy of how our visual system works under natural conditions. In this respect, our study gives interesting evidence that is in line with recent works showing evoked responses to freely gazed complex stimuli (Graupner et al., 2007; Luo et al., 2009; Ossandon et al., 2010; Kamienkowski et al., 2012; Kaunitz et al., 2014). In addition, it paves the way for new studies and experimental paradigms dealing with visual responses and cognitive processes in more ecological conditions, using magic tricks 
and/or responses to freely gazed videos. 


\section{References}

Ahmadi M, Quian Quiroga R (2013) Automatic denoising of single-trial evoked potentials. Neuroimage 66:672-680.

Binet A, Nichols M (1896) Psychology of prestidigitation. publisher not identified.

Blake R, Logothetis NK (2002) Visual competition. Nature Reviews Neuroscience 3:13-21. Brainard DH (1997) The psychophysics toolbox. Spat Vis (NETHERLANDS) 10:433-436.

Cui J, Otero-Millan J, Macknik SL, King M, Martinez-Conde S (2011) Social misdirection fails to enhance a magic illusion. Front Hum Neurosci (Switzerland) 5:103.

Danek AH, Öllinger M, Fraps T, Grothe B, Flanagin VL (2015) An fMRI investigation of expectation violation in magic tricks. Frontiers in Psychology 6:.

Donchin E (1981) Surprise!... surprise? Psychophysiology 18:493-513.

Freeman W, Quian Quiroga R (2012) Imaging brain function with EEG: Advanced temporal and spatial analysis of electroencephalographic signals. Springer.

Graupner ST, Velichkovsky BM, Pannasch S, Marx J (2007) Surprise, surprise: Two distinct components in the visually evoked distractor effect. Psychophysiology (United States) 44:251261.

Gregory RL (1973) The confounded eye. Illusion in Nature and Art 49-96.

Helmholtz H (1878a) Selected writings of hermann von helmholtz (R. kahl, ed.).

Helmholtz H (1878b) The facts of perception. Selected Writings of Hermann Von Helmholtz $366-408$.

Jastrow J (1897) Magic Stage Illusions and Scientific Diversions, Including Trick Photography . Johansson P, Hall L, Sikstrom S, Olsson A (2005) Failure to detect mismatches between intention and outcome in a simple decision task. Science (United States) 310:116-119.

Kamienkowski JE, Ison MJ, Quian Quiroga R, Sigman M (2012) Fixation-related potentials in visual search: A combined EEG and eye tracking study. J Vis (United States) 12:4. Kanwisher N (2001) Neural events and perceptual awareness. Cognition 79:89-113. 
Kaunitz LN, Kamienkowski JE, Varatharajah A, Sigman M, Quiroga RQ, Ison MJ (2014)

Looking for a face in the crowd: Fixation-related potentials in an eye-movement visual search task. Neuroimage (United States) 89:297-305.

Kuhn G, Caffaratti H, Teszka R, Rensink R (2014) A psychologically-based taxonomy of misdirection. Frontiers in Psychology .

Kuhn G, Land MF (2006a) There's more to magic than meets the eye. Current Biology 16:R950R951.

Kuhn G, Findlay JM (2010) Misdirection, attention and awareness: Inattentional blindness reveals temporal relationship between eye movements and visual awareness. Q J Exp Psychol (Hove) (England) 63:136-146.

Kuhn G, Land MF (2006b) There's more to magic than meets the eye. Curr Biol (England) 16:R950-1.

Kuhn G, Kourkoulou A, Leekam SR (2010) How magic changes our expectations about autism. Psychol Sci (United States) 21:1487-1493.

Kuhn G, Amlani AA, Rensink RA (2008) Towards a science of magic. Trends Cogn Sci (England) 12:349-354.

Lamont P, Wiseman R (2005) Magic in theory: An introduction to the theoretical and psychological elements of conjuring. Univ of Hertfordshire Press.

Lamont P, Henderson JM (2009) More attention and greater awareness in the scientific study of magic. Nat Rev Neurosci (England) 10:241; author reply 241-c1. Epub 2009 Jan 29.

Logothetis NK (1998) Single units and conscious vision. Philos Trans R Soc Lond B Biol Sci (ENGLAND) 353:1801-1818.

Luck SJ, Kappenman ES (2011) The oxford handbook of event-related potential components. Oxford university press.

Luo A, Parra L, Sajda P (2009) We find before we look: Neural signatures of target detection preceding saccades during visual search. Journal of Vision 9:1207-1207.

Macknik SL, King M, Randi J, Robbins A (2008) Attention and awareness in stage magic: Turning tricks into research. Nature Reviews Neuroscience 9:871-879. 
Mark Wilson \& Earl Nelson (1979) The chop cup book. Los Angeles, CA: .

Martinez-Conde S, Macknik SL (2008) Magic and the brain. Sci Am (United States) 299:72-79. Navajas J, Ahmadi M, Quian Quiroga R (2013) Uncovering the mechanisms of conscious face perception: A single-trial study of the n170 responses. J Neurosci (United States) 33:1337-1343. Olson JA, Amlani AA, Rensink RA (2012) Perceptual and cognitive characteristics of common playing cards. Perception (England) 41:268-286.

Ossandon JP, Helo AV, Montefusco-Siegmund R, Maldonado PE (2010) Superposition model predicts EEG occipital activity during free viewing of natural scenes. J Neurosci (United States) $30: 4787-4795$.

Otero-Millan J, Macknik SL, Robbins A, Martinez-Conde S (2011) Stronger misdirection in curved than in straight motion. Front Hum Neurosci (Switzerland) 5:133.

Parris BA, Kuhn G, Mizon GA, Benattayallah A, Hodgson TL (2009) Imaging the impossible: An fMRI study of impossible causal relationships in magic tricks. Neuroimage 45:1033-1039. Picton TW (1992) The P300 wave of the human event-related potential. J Clin Neurophysiol (UNITED STATES) 9:456-479.

Polich J (2007) Updating P300: An integrative theory of P3a and P3b. Clin Neurophysiol (Netherlands) 118:2128-2148.

Quian Quiroga R (2016) Magic and cognitive neuroscience. Current Biology 26:R1-R5. Quian Quiroga R (2000) Obtaining single stimulus evoked potentials with wavelet denoising. Physica D 145:278-292.

Quian Quiroga R, Kraskov A, Mormann F, Fried I, Koch C (2014) Single-cell responses to face adaptation in the human medial temporal lobe. Neuron 84:363-369.

Quian Quiroga R, Mukamel R, Isham EA, Malach R, Fried I (2008) Human single-neuron responses at the threshold of conscious recognition. Proc Natl Acad Sci U S A (United States) 105:3599-3604.

Raz A, Zigman P (2001) Using magic as a vehicle to elucidate attention. In: eLS Using magic as a vehicle to elucidate attention. John Wiley \& Sons, Ltd. 
Rieiro H, Martinez-Conde S, Macknik SL (2013) Perceptual elements in penn \& teller's “Cups and balls" magic trick. PeerJ 1:e19.

Shalom DE, de Sousa Serro, Maximiliano G, Giaconia M, Martinez LM, Rieznik A, Sigman M (2013) Choosing in freedom or forced to choose? introspective blindness to psychological forcing in stage-magic. PloS One 8:e58254.

Sutton S, Braren M, Zubin J, John ER (1965) Evoked-potential correlates of stimulus uncertainty. Science (UNITED STATES) 150:1187-1188.

Triplett N (1900) The psychology of conjuring deceptions. Am J Psychol 11:439-510.

Walter WG, Cooper R, Aldridge VJ, McCallum WC, Winter AL (1964) Contingent negative variation: An electric sign of sensorimotor association and expectancy in the human brain. Nature (ENGLAND) 203:380-384.

Wilson M, Nelson E (1979) The chop cup book. Los Angeles, CA: .

Legends

Figure 1. General structure of a trial. (A) Direct Load: The ball was placed inside the cup (1). The cup was turned upside down but covered with the right hand (2). The cup remained shortly in the air upside down while the magician removed the right hand, apparently with the ball (because if the ball was still inside the cup, it would fall) (3). The cup was placed on the table (4). The video was then paused, waiting for the subject's response (guessing whether the ball was in the cup or in the hand). After a short delay following the subject's response (orange line), the video was restarted, revealing the content of the cup $\left(5,5^{\prime}\right)$. The magician then introduced the ball in the cup again starting a new trial. (B) In Experiment 2, 'Direct Load' trials were interleaved with 'Indirect Load' trials, in which the movement of taking the ball in the hand (2-3) was performed under the table. 
Figure 2. Behavioral results. Average percentage of predictions that the ball remain in the cup ('cup responses'), for Experiment 1 (left) and for Experiment 2 (right). In Experiment 1, the number of 'cup responses' wasn't significantly different between Block 1 and Block 2. In Experiment 2 the percentage of 'cup responses' was significantly different depending on whether there was a Direct Load performed (movement done on top of the table) or not (movement done under the table, 'Indirect Load').

Figure 3. Grand average evoked responses for Experiment 1. In Block 1 the ball appeared in the cup ('Cup_Direct-Load' trials) $50 \%$ of the time and the other $50 \%$ was retained in the hand ('Hand_Direct-Load' trials), whereas in Block 2 the proportion of cup/hand trials was $30 \% / 70 \%$. In all conditions there was a slow negative shift preceding stimulus onset $(\mathrm{CNV})$ reflecting expectation, and a $\mathrm{P} 3$ response, which was larger and immediately preceded by an N2 response for the infrequent 'Cup_DirectLoad trials' in Block 2. Time zero corresponds to stimulus onset (the time where the content of the cup was revealed). Shaded areas around mean values show SEM.

Figure 4. (A) Grand average evoked responses for Experiment 2. Responses are separated according to: the type of trial (ball appearing in the cup or retained in the hand) and condition (presentation preceded by the performance of a magic trick or not, that is 'Direct Load' (DL) vs 'Indirect Load' (IL)). 'Cup trials' in the 'Direct Load' and 'Indirect Load' conditions elicited larger and earlier responses compared to the 'Hand trials'. For the 'cup trials', the responses had a later onset under the 'Direct Load' condition. Notations are the same as in the previous figure. (B) Grand average of the P3 peak latencies for the cup responses of Experiment 2, separated according to the 
condition ('Direct Load' (DL), 'Indirect Load' (IL)) and type of trial ('correct', 'incorrect'). Both for the correct and incorrect trials, the 'cup responses' appeared later for the 'Direct Load' condition. The stars shown indicate which of these differences where statistically significant. 